\title{
PHASE TRANSFORMATIONS AND INHERITED ODFS : IMPLICATIONS FOR PETROPHYSICAL PROPERTIES
}

\author{
D. MAINPRICE \\ Laboratoire de Tectonophysique \\ Université de Montpellier II, 34095 Montpellier Cedex 05 - France
}

\author{
M. HUMBERT and F. WAGNER \\ Laboratoire de Métallurgie des Matériaux Polycrystallins \\ Université de Metz, 57045 Metz Cedex - France
}

\section{INTRODUCTION}

Seismic discontinuities in the Earth's mantle have been interpreted as phase transformations (1). Little attention has been paid to the three dimensional nature of seismic properties of regions undergoing phase transformation or the anisotropy of the petrophysical properties of such regions. Recent progress in anisotropic seismic tomography (2) reveals the need for a better understanding of the anisotropic rock properties. Potentially the knowledge of anisotropic properties will add a futher constraint for the development of more realistic large scale geodynamic models.

\section{PHASE TRANSFORMATIONS}

The mechanisms phase transformation can be complex, we can to a first approximation classify the mechanisms into two classes.

1. Nucleation \& Growth, a mechanism which depends on a nucleation event and diffusion controlled growth process. The nucleation is usually hetereogeneous on imperfections. Depending on the anisotropy of crystal structure and the thermodynamic conditions this mechanism may or may not result in an orientation relationship between "old" and "new" phases.

2. Martensitic mechanism is a diffusionaless transformation. The crystal structure of the "old" phase is sheared in systematic coordinated manner such that any atom is moved by less than one atomic spacing. Martensitic transformation results in changes of crystal structure, but not composition. Such transformation always produce a fixed orientation relationship between the "old" and "new" phase.

\section{DESCRIPTION OF ORIENTATION RELATIONSHIPS}

It is traditional in quantative texture analysis to describe the orientations of an ensemble of grains by the Orientation Distribution Function (ODF) (3). The orientation of an individual grain (crystal) may be defined by three Euler angles $(\varphi 1, \phi, \varphi 2)$ which 
describe the rotation between the crystal and specimen reference frames. In a similar manner one can define the orientation $(\Delta g)$ of the "old" phase reference with respect to "new" phase (fig.1). Using such a description of the orientation relationships it is easy to show that the orientation of the new phase is given by

$$
\left(g_{n}^{n e w}\right)=\Delta g \cdot s_{n}^{\text {old }} \cdot g \text { old }
$$

where $s_{n}^{\text {old }}$ is the $n$ symmetry operations of the old phase and gold is the "old" phase orientation. Using such an approach we have calculated the ODF of the "new" phase using the ODF of the "old" phase, the symmetry operations and the know orientation relationships $(\Delta \mathrm{g})$.

\section{CALCULATION OF PETROPHYSICAL PROPERTIES}

Given the appropriate tensor transformation law (4) we can rotate the petrophysical constants into their orientation in sample coordinates. For the case of triclinic crystal and specimen symmetry the average polycrystal tensor $(\overline{\mathbf{T}})$ can be determined by integration as

$$
\begin{gathered}
\bar{T}=\int T(g) \cdot f(g) \cdot d g \\
d g=\frac{1}{82^{2}} \sin \Phi d \varphi_{1} d \Phi d \varphi_{2}
\end{gathered}
$$

where $T(g)$ is the tensor in sample coordinates and $f(g)$ the ODF. The familiar Voigt, Reuss or Voigt-Reuss-Hill averages may be calculated in this manner.

\section{ALPHA-BETA QUARTZ TRANSITION}

The alpha-beta quartz transition is a good test case for the method presented here because all the petrophysical constants have been determined. We have used the ODF of an alpha-quartz polycrystal (fig.2) quartzite GRAN133 (5) which is known to have experienced $\beta$-quartz stability conditions (granulite metamorphic facies).

For the extraction of hydrothermal energy, thermal cracking of the source region is an important process for producing permeability in granites and quartzites. The anisotropy of thermal expansion, elastic properties and the temperature change are the important parameters which determine the degree and orientation of thermal cracks. The thermal expansion of a quartz single crystal and polycrystal GRAN133 (fig.3) have been calculated using the data of Skinner (6). The minimum thermal expansion occurs parallel to the c-axes. The smoothness of the distribution of thermal expansion is due to the second order nature of this tensor property.

We have undertaken a simimar calculation for the compressional seismic velocity (Vp) as a function of temperature for GRAN133 (fig. 4) using the single crystal elastic constants of McSkimin et al. (7), Zubor and Firsova (8), Kammer et al. (9) to cover the temperature range. The calculated $\mathrm{Vp}$ can be compared with experimental determination by Kern (10) on a quartzite with a weak texture. The calculations agree well with the experimental results except that the minimum velocity, associated with the 
transition is displaced by $80^{\circ} \mathrm{C}$. The shift in the experimental minimum is due to internal stresses generated by the anisotropic thermal expansion. The differential stresses cause a shift of the transition temperature (11).

\section{OLIVINE-SPINEL TRANSITION}

Various models have been proposed for the olivine-> $\beta$-spinel transition in recent years. The transition is thought to be responsible for the major seismic discontinuity in compressional $(\mathrm{Vp})$ and shear wave (Vs) velocities at $400 \mathrm{~km}$ depth in the Earth's Mantle. As an illustration of the potential of the method described here we will model the transition using the crystallographic relationships for $\Delta \mathrm{g}$ proposed by Madon and Poirier (12) from which

\section{(1 0 0) olivine $/ /\left(\begin{array}{lll}1 & \overline{1} & 0\end{array}\right) \beta$-spinel \\ $\left[\begin{array}{lll}0 & 0 & 1\end{array}\right]$ olivine // [ [ $\left.\begin{array}{lll}0 & 0 & 1\end{array}\right]$-spinel}

For the ODF of the "old" or olivine phase we have used an ODF calculated from a kimberlite nodule (Bulfontein, South Africa) extracted from a depth of $140 \mathrm{~km}$ in the Upper mantle (13). The calculated pole figures for the $\beta$-spinel (fig. 5) show the alignement of [001] olivine and [001] spinel. The calculated Vp for olivine show $a$ maximum which corresponds to maximum of [100] axes (the fast Vp direction), similarly the calculated $\mathrm{Vp}$ for $\beta$-spinel shows a maximum which corresponds to a maximum of [010] axes. Note that the maximum velocities in the olivine and spinel polycrystals are not parallel but rotated by $90^{\circ}$, indicating a fundamental change in the orientation of the seismic anisotropy at the transition.

The composition of the Mantle is currently the subject of much speculation. At the $400 \mathrm{~km}$ depth the composition has been estimated to be either $70 \%$ olivine (1) or $40 \%$ olivine (14). Seismic observations suggest that velocity jump $(\Delta \mathrm{Vp})$ at $400 \mathrm{~km}$ for $\mathrm{Vp}$ is

$$
3.5 \%<\left(\frac{\Delta V p}{V p}\right) \text { obs }<6 \%
$$

according to Montagner and Anderson (2). If the transition occurs over a small depth interval ( less than $10 \mathrm{~km}$ ) (15) we can assume that only phase transformation contributing to $\Delta \mathrm{Vp}$ is the olivine-> $\beta$-spinel. It is reasonable to estimate the volume fraction of olivine $\left(\mathrm{X}_{\mathrm{Ol}}\right)$ in the transition zone by

$$
\mathrm{X}_{\mathrm{ol}}=\frac{(\Delta \mathrm{Vp} / \mathrm{Vp}) \text { obs }}{(\Delta \mathrm{Vp} / \mathrm{Vp}) \alpha-\beta}
$$

where $(\Delta V p / V p)$ obs is given by Montagner and Anderson (2) and $(\Delta V p / V p) \alpha-\beta$ is the velocity change for a pure olivine aggregate transforming to $\beta$-spinel. Using such an approach will give values of between $56 \%<\mathrm{X}_{\mathrm{ol}}<96 \%$ for propagation directions along $\mathrm{X}$ in fig. 5 or $19 \%<\mathrm{X}_{\mathrm{Ol}}<33 \%$ for propagation along $\mathrm{Z}$. We suggest that it important to take into account the propagation direction when undertaking such evaluations. 


\section{CONCLUSIONS}

1. A quantative calculation of the petrophysical properties of a transformed polycrystal is possible.

2. The seismic properties "above" and "below" the phase transformation (e.g. $400 \mathrm{~km}$, olivine-spinel) can be used to characterize an interface or reflector.

3. Further constaints on the Earth's Mantle composition may be possible with ;

a) control of the propagation direction in seismic data

b) crystallographic characterization of the transformation under Mantle conditions (low stress, high temperature and pressure).

\section{ACKNOWLEGEMENTS}

Finanicial support to D.M. from the CNRS/INSU DBT-Geodymamics program is gratefully acknowledged.

\section{REFERENCES}

1. Ringwood, A.E. Compostion and Petrology of the Earth's Mantle p.618 (McGrawHill, New York, 1975).

2. Montagner, J.P. \& Anderson, D.L. Phys.Earth Planet.Int. 58, $205-227$ (1989).

3. Bunge, H.J. Texture analysis in Materials Science p.593 (Butterworths, London, 1982).

4. Nye, J.F. Physical properties of crystals. Their representation by tensors and matrices p.322 (Clarendon Press, Oxford, 1957).

5. Schmid, S.M., Casey, M. \& Starkey, J. Tectonophysics 78, 101-117 (1981).

6. Skinner, B.J. in Handbook of Physical Constants (ed. S.P. Clark, Jr.) 75-96 (Geol. Soc. Am. Bull., 1966).

7. McSkimin, H.J., Anreatch, J.R. \& Thurston, R.N. J. Appl. Phys. 36, 1624-1632 (1965).

8. Zubov, V.G. \& Firsova, M.M. Soviet Phys.-Cryst., English Transl. 7, 374-376 (1962).

9. Krammer, E.W., Pardue, T.E. \& Frissel, H.F. J. of Appled Phys. 19, 265-270 (1948).

10. Kern, H. Phys. Chem. Mineral. 4, 161-171 (1979).

11. Coe, R.S. \& Paterson, M.S. J. Geophys. Res. 74, $4921-4948$ (1969).

12. Madon, M. \& Poirier, J.P. Phys. Earth Plant. Int. 33, 31-44 (1983).

13. Boullier, A.M. Structure des peridotites enclaves dans les kimberlites d'Afrique de Sud (Université de Nantes, 1975).

14. Anderson, D.L. \& Bass, J.D. Nature 320, 321-328 (1986).

15. Bina, C.R. \& Wood, B.J. J.Geophys.Res. 92, 4853-4866 (1987). 
Misorientation $\Delta \mathrm{g}$ between olivine \& beta-spinel

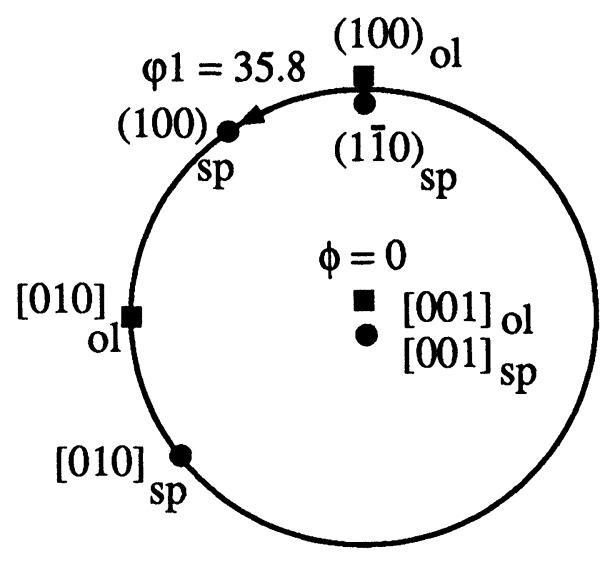
$\Delta \mathrm{g}=(\varphi 1, \phi, \varphi 2)=35.8,0,0$

FIGURE 1. Euler angle description of misorientation between phases

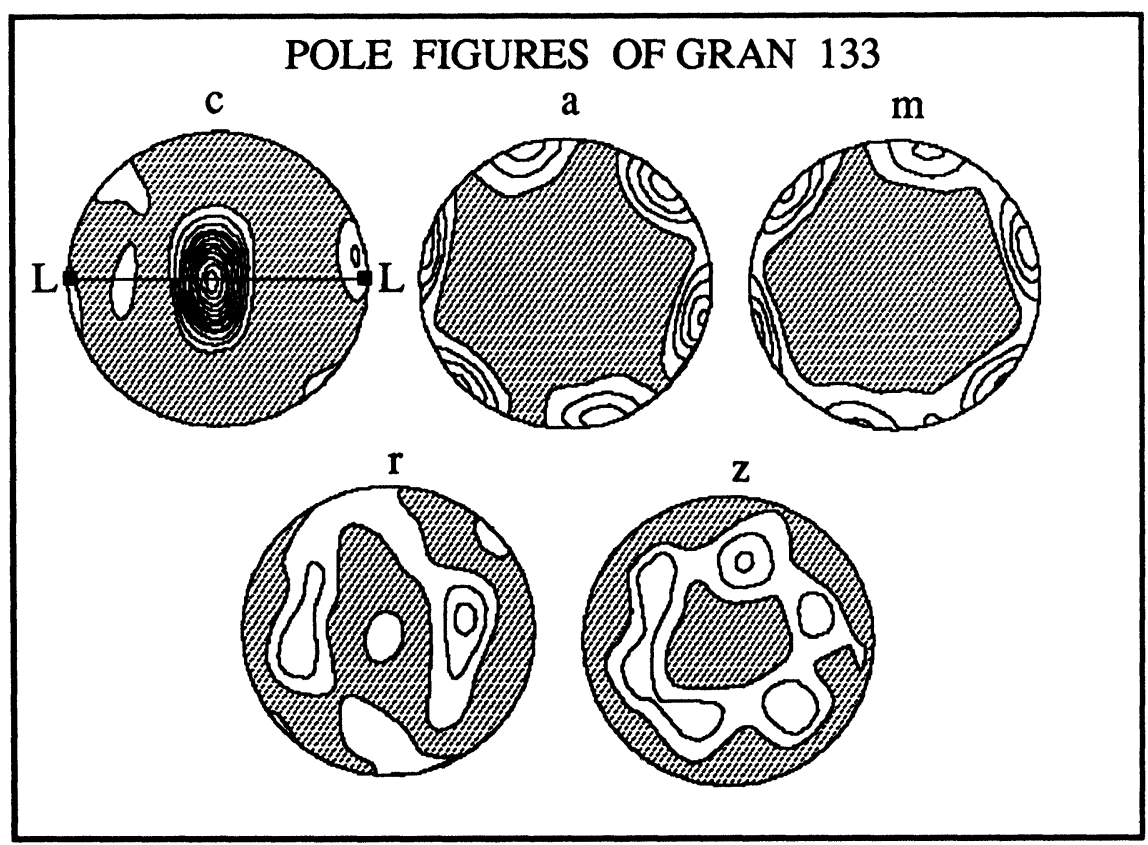

FIGURE 2. Polefigures of quartzite GRAN 133 recalculated from ODF 
THERMAL EXPANSION OF QUARTZ AND GRAN 133
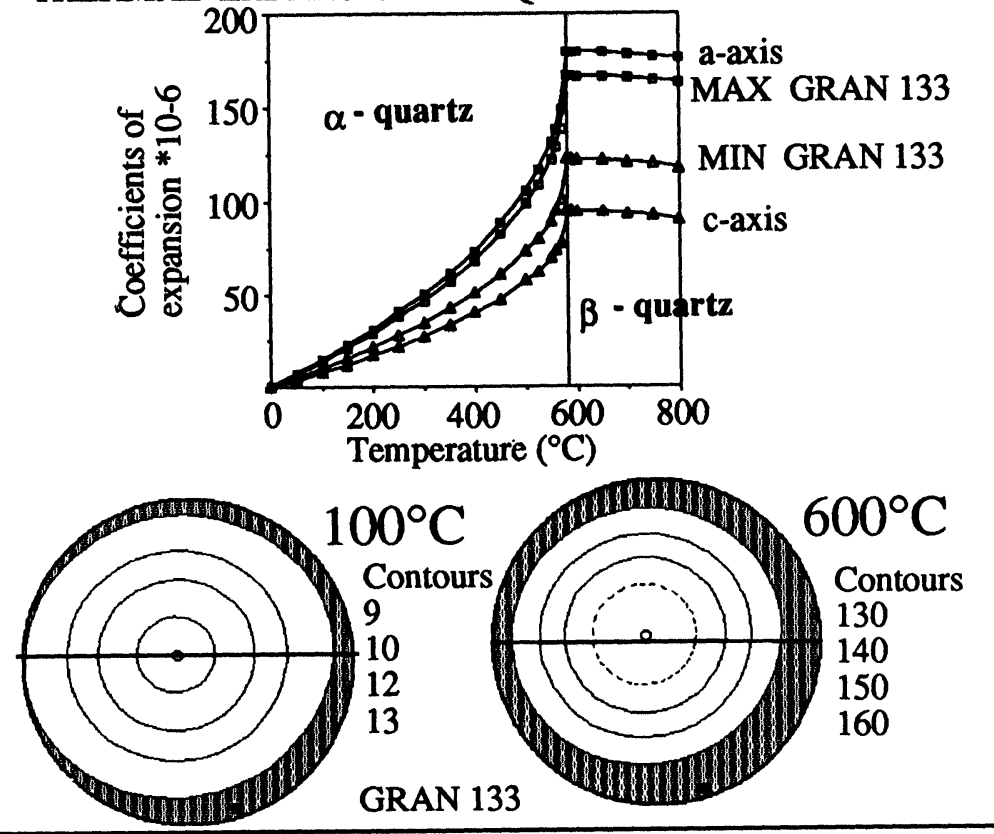

FIGURE 3. Thermal expansion for GRAN 133 calculated from ODF

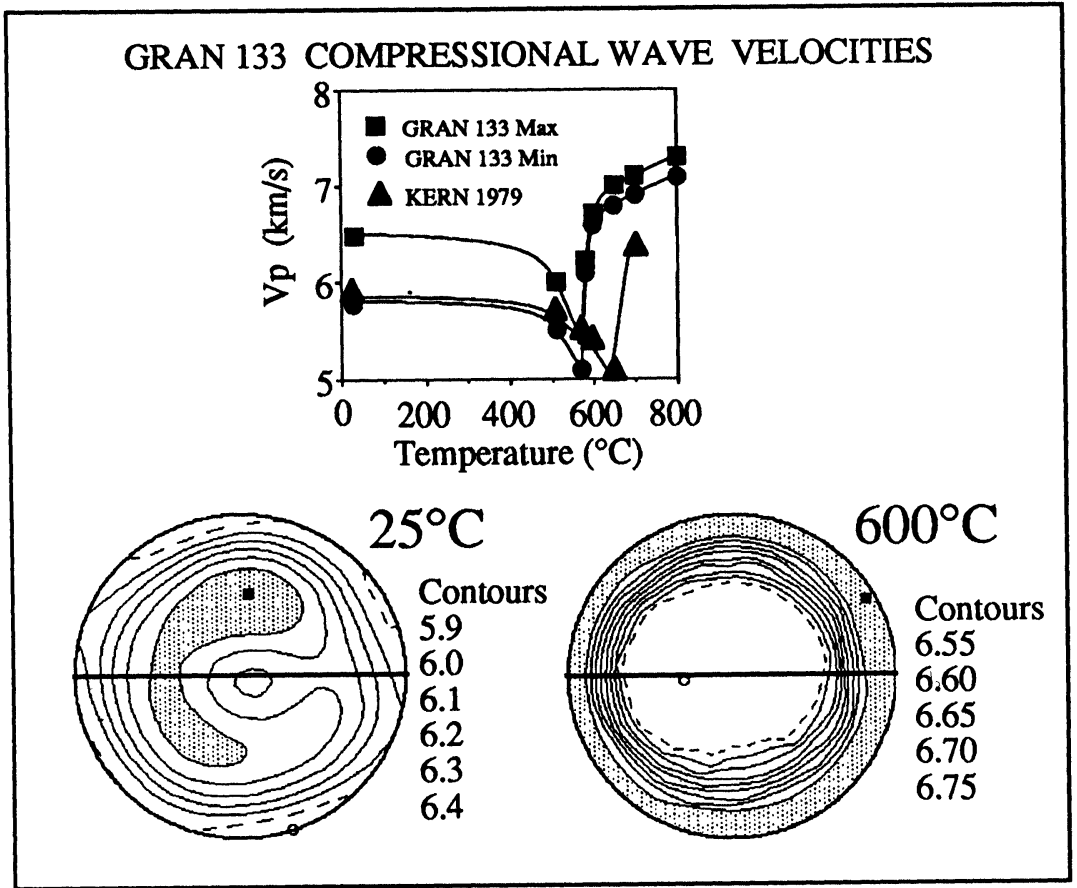

FIGURE 4. Compressional wave velocities for GRAN 133 calculated from ODF 


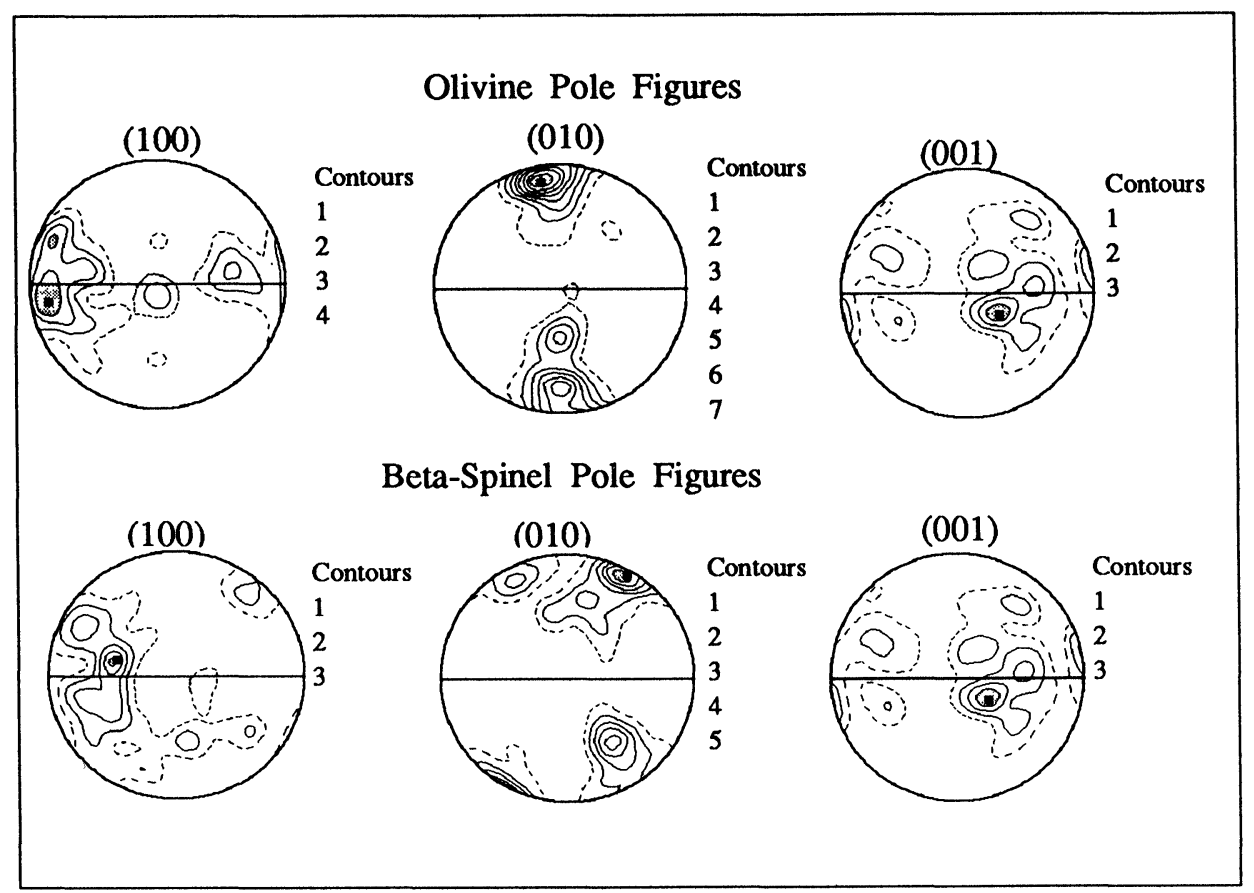

FIGURE 5. Olivine and Beta-Spinel pole

figures recalculated from ODF

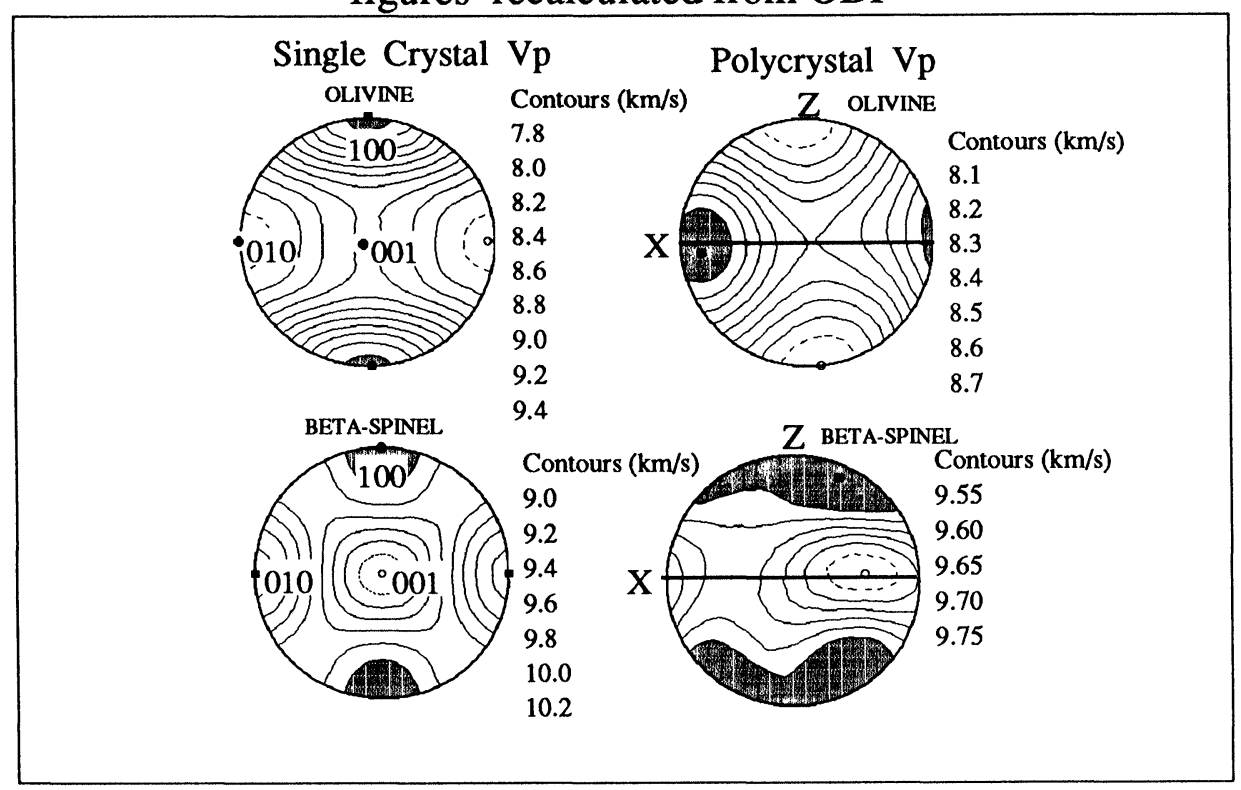

FIGURE 6. Compressional wave velocities for single crystal and polycrystalline olivine and beta-spinel 